\title{
2.7 RADIO OBSERVATIONS OF THE CRAB PULSAR
}

\author{
AT 408, 240 AND $151 \mathrm{MHz}$
}

\author{
RUDOLF E. SCHÖNHARDT \\ University of Manchester, Nuffield Radio Astronomy Laboratories, \\ Jodrell Bank, U.K.
}

The radio pulses from the Crab pulsar have been observed at Jodrell Bank on several days in June, July and August 1970 at frequencies of $408 \mathrm{MHz}, 240 \mathrm{MHz}$ and 151 $\mathrm{MHz}$. The integrated pulse shapes and the linear polarization of the average pulses have been investigated.

Most of the measurements at $408 \mathrm{MHz}$ were in general agreement with earlier observations performed at Arecibo (Campbell et al., 1970). The receiver bandwidth used at Jodrell Bank was $13 \mathrm{kHz}$. The linear polarization in the main pulse and interpulse is roughly $18 \%$ and $10 \%$ respectively. The degree of linear polarization in the precursor is near $100 \%$, but may be somewhat variable. The position angle of the linearly polarized radiation is fairly constant throughout the precursor; any possible swing is less than about $15^{\circ}$ across the pulse width.

The interesting question of a possible swing of the polarization angle in the main pulse was investigated. Four integrations of about 1.5 hours integration time each were obtained on different days in June, July and August. In three of these integrations a swing of roughly the same rate and of the same direction seemed to be visible. As the signal to noise ratios in these integrations were not very satisfactory, all the four integrations were added together, yielding a total integration time of 6.7 hours (see Figure 1). Within the errors calculated from the rms noise one obtains a swing rate of $100^{\circ}\left( \pm 40^{\circ}\right)$ per millisecond. This rate is in agreement with the optical swing rate near the peak of the optical pulse of about $90^{\circ}$ per millisecond (Wampler et al., 1969). Unfortunately the question of the direction of the rotation in the radio pulse cannot yet be settled.

In some of the integrations a small pulse was detected, occurring $5.2( \pm 0.3) \mathrm{msec}$ after the main pulse. This 'postcursor' was detected at $408 \mathrm{MHz}$ and $240 \mathrm{MHz}$ (Figure 2). At both these frequencies its energy was roughly $7.5 \%( \pm 2.5 \%)$ of the sum of the energies of the main pulse and precursor.

At $240 \mathrm{MHz}$ the degree of linear polarization in the precursor is about $50 \%$ (Figure 3a), but it seems to vary as seen by comparing Figure $3 \mathrm{~b}$ and Figure 3c.

At $151 \mathrm{MHz}$ the stronger pulse could no longer be resolved into a precursor and main pulse, but its linear polarization of the order of $15 \%$ to $20 \%$ could still be clearly detected.

\section{References}

Campbell, D. B., Heiles, C., and Rankin, J. M.: 1970, Nature 225, 527.

Wampler, E. J., Scargle, J. D., and Miller, J. S.: 1969, Astrophys. J. Letters 157, L1.

Davies and Smith (eds.), The Crab Nebula, 110-113. All Rights Reserved.

Copyright (C) 1971 by the IAU. 


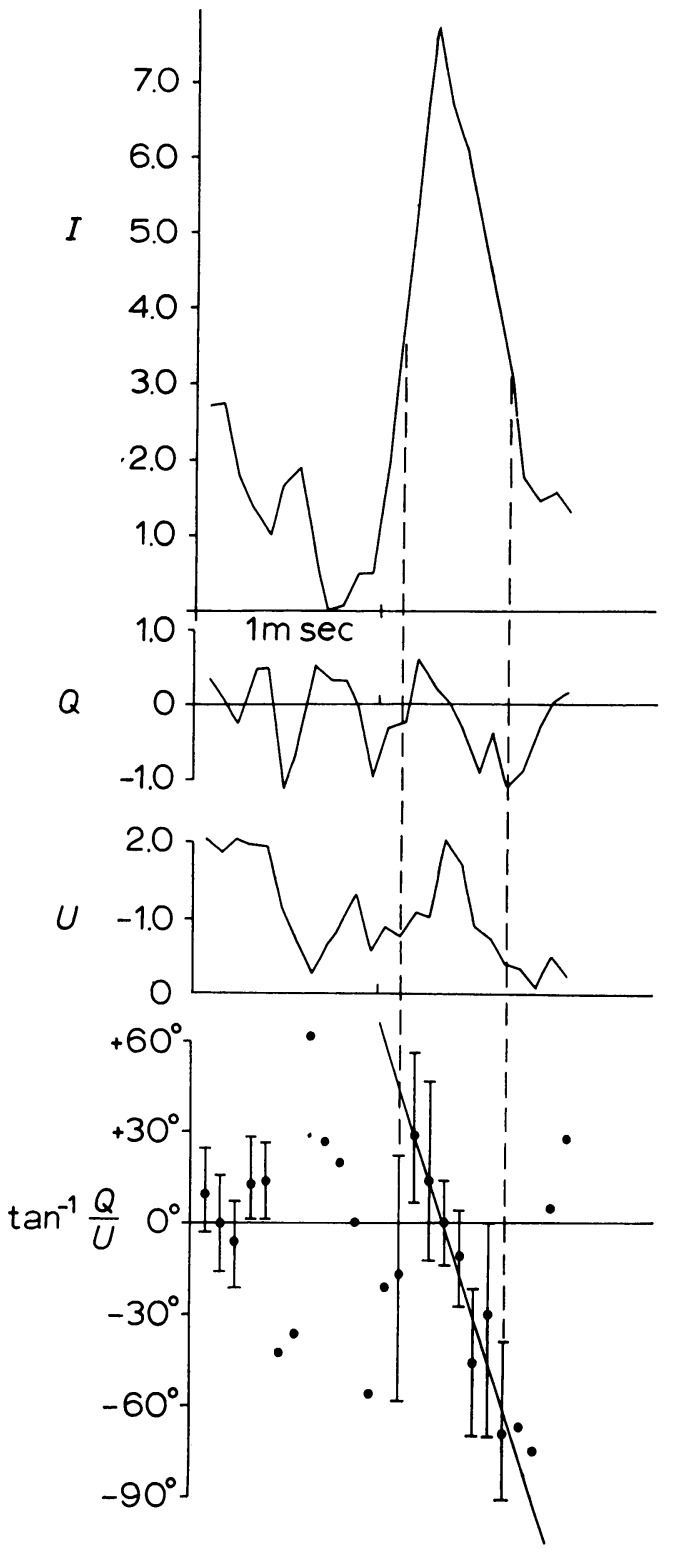

Fig. 1. Main Pulse at $408 \mathrm{MHz}$; integration time $=6 \mathrm{~h} 45 \mathrm{~min}$.

\section{Discussion}

C. Heiles: I would like to report a new result obtained by Rankin and me at Arecibo concerning the average pulse shape at $606 \mathrm{MHz}$, namely that in two days $(6 \mathrm{~h})$ of integration we were unable to see the precursor. If it was as strong in total intensity relative to the main pulse as it is at $430 \mathrm{MHz}$ its amplitude would have been more than 10 times the noise on our average. Our null detection is possibly the result of our feed, which is linearly polarised, being unfavourably oriented with respect to the position angle of the linearly polarised precursor. Assuming that this is the case, we are able 


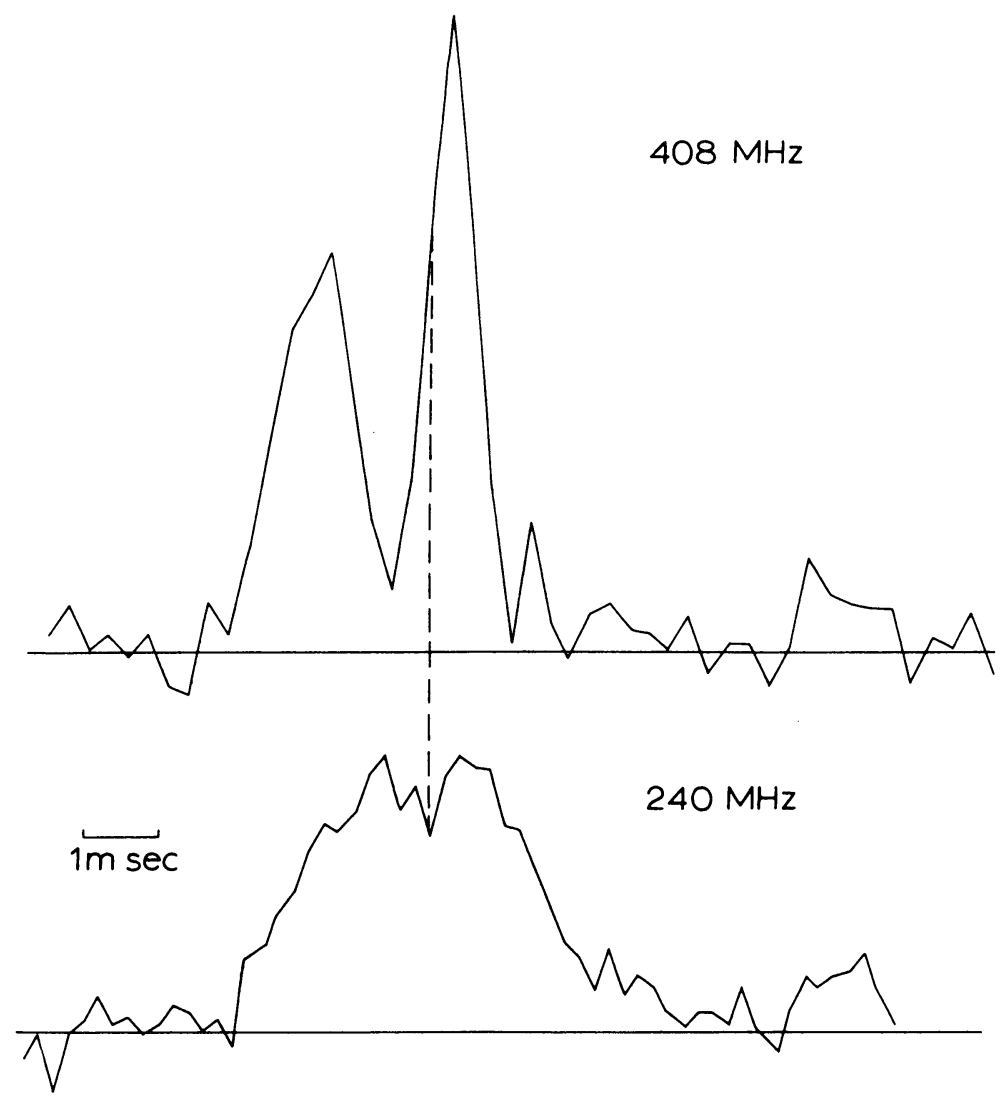

Fig. 2. The postcursor at $408 \mathrm{MHz}$ and $240 \mathrm{MHz}$.

to say that the relative intensity of the precursor at $606 \mathrm{MHz}$ is less than $60 \%$ of its value at $430 \mathrm{MHz}$.

J. Rankin: Although we observe a good deal of emission between the main pulse and interpulse at frequencies between 430 and $196.5 \mathrm{MHz}$, we have never noted a specific short component in the average of several hundred thousand pulses.

$M$. M. Komesaroff: Was the swing in position angle of the polarisation of the radio emission of the main Crab pulsar pulse as seen by Mr. Schönhardt similar to that previously reported as seen optically?

R. Schönhardt: I may remark that in our integrations of high resolution the broadening due to dispersion within the bandwidth was $90 \mu \mathrm{sec}$. For our bandwidth of $13 \mathrm{kHz}$, the inverse of the bandwidth is $77 \mu$ sec.

A.T. Moffet: Arecibo results show no swing of position angle within the main pulse of the Crab pulsar. Can this be reconciled with the Jodrell results?

F. D. Drake: At Arecibo it has not been possible to measure the rotation of the position angle of the linear polarisation of the main pulse due to inadequate time resolution. Time resolution is limited by dispersion effects, which require one to reduce bandwidth as much as possible. However, it is only worthwhile to reduce the bandwidth to the value whose reciprocal is equal to the time smearing introduced by dispersion over that bandwidth. At $430 \mathrm{MHz}$ this limiting time resolution is $130 \mu \mathrm{sec}$ in NP 0532. With this resolution one can only resolve the main pulse into two intervals. 
(a)

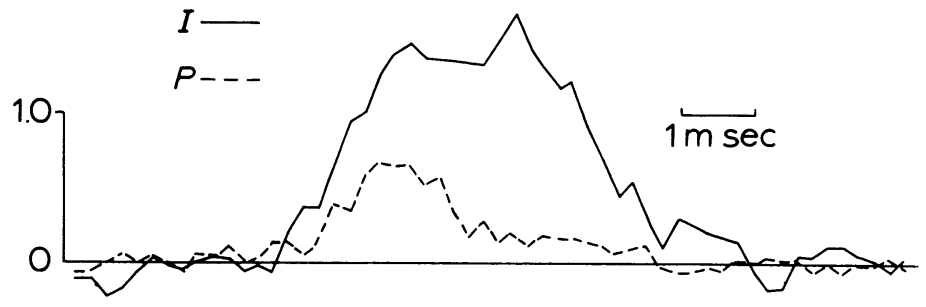

(b)
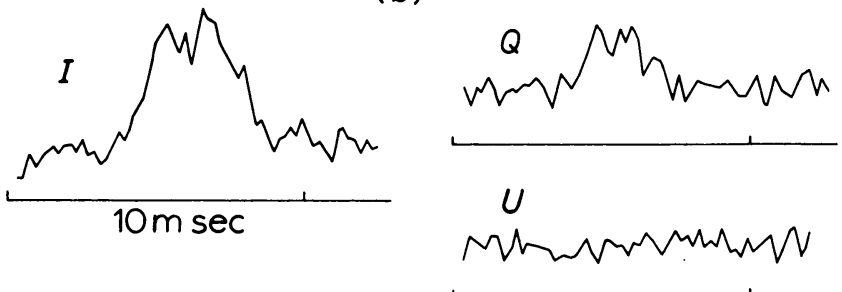

(c)
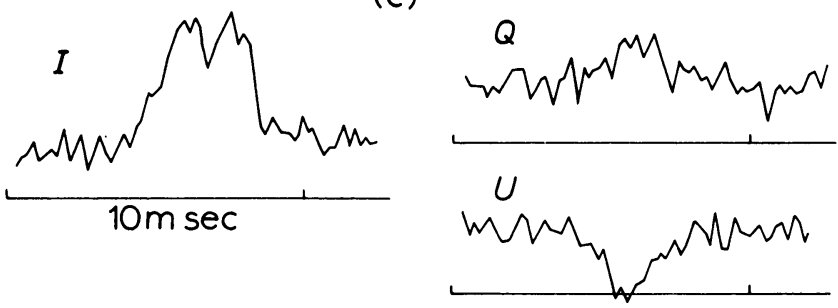

Fig. 3. (a) $240 \mathrm{MHz}$, main pulse and precursor ( $I$ and $P$ ). (b) $240 \mathrm{MHz}$, main pulse and precursor $(I, Q$ and $U$ ). (c) $240 \mathrm{MHz}$, main pulse and precursor $(I, Q$ and $U$ ), at a later date.

With these few intervals, the small intrinsic polarisation, and the small signal/noise ratio, no significant measurement of polarisation rotation is possible.

In view of the fact that the intrinsic dispersion limit on time resolution also applies to Jodrell Bank data, it is hard to understand how a definitive polarisation measurement could be made. Multichannel radiometers are required to make this measurement properly. 